\title{
Sentiment Analysis of Amazon Products Using Ensemble Machine Learning Algorithm
}

\author{
Jayakumar Sadhasivam \\ Department of Information Technology and Engineering \\ School of Information Technology and Engineering \\ Vellore Institute of Technology, Vellore, India \\ Corresponding author: jayakumars@vit.ac.in \\ Ramesh Babu Kalivaradhan \\ Department of Computer Sciences and Engineering \\ School of Computer Sciences and Engineering \\ Vellore Institute of Technology, Vellore, India \\ E-mail: krameshbabu@vit.ac.in
}

(Received October 15, 2018; Accepted February 5, 2019)

\begin{abstract}
In recent years, Sentimental Analysis is used in all online product firms. The number of users using the particular product has increased which makes the industry to improvise the characteristics of the product. These days, many users who are using websites, blogs, online shopping tends to review the products they used. These reviews were taken into consideration by other users during their search for products. Hence the industry has found the root of delivering the correct product searched by the user based on the reviews of the users using the concept of sentimental analysis. Sentimental Analysis is the concept of data analysis where the collections of reviews are taken into consideration, and those reviews are analyzed, processed and recommended to the user. The reviews given are longer and which consist of a few paragraphs of content. In this paper, the dataset is collected from the official product sites. Initially, these reviews must be pre-processed in order to remove the unwanted data's such as stop words, be verbs, punctuations, and conjunctions. Once, the pre-processing is over the trained dataset is classified using Naive Bayes and SVM algorithm. These existing algorithms provided the accuracy which is not worth enough. Hence, an ensemble approach has been applied to enhance the accuracy of the given reviews. An ensemble is a classification approach by combining two or more algorithms and calculate the mode value based on the vote reference for every algorithm which is used. In this paper, Naive Bayes, SVM, and Ensemble algorithm are combined. We proposed an Ensemble method that helps in providing better accuracy than the current existing algorithm. Once the accuracy is calculated, based on the reviews, the particular product is recommended for the user.
\end{abstract}

Keywords- Machine learning, Naïve Bayes, SVM, Sentimental analysis, Ensemble method.

\section{Introduction}

Extracting the public opinion from the text reviews from various websites through computational methods and analyzing becoming a trending research study these days. A set of root words based on traditional and slag languages which obtained are used in those researches. These researchers are providing an optimizing approach to gathering the relevant data/text from the Internet and help in processing the data. Sentiment expressions are being recognized as through these approaches. Generally, the review datasets are classified as supervised and unsupervised datasets. In supervised learning, researchers have a set of labeled data, meaning that the researchers have the values of the inputs and the outputs. What do want to achieve with machine learning is to find the exact relationship between them, what we usually call the model in math? There are many different algorithms in machine learning that enable us to obtain a model of the data. The objective that the 
International Journal of Mathematical, Engineering and Management Sciences

Vol. 4, No. 2, 508-520, 2019

https://dx.doi.org/10.33889/IJMEMS.2019.4.2-041

researchers seek, and how we can use machine learning, is to predict the output given a new input, once we know the model. In unsupervised learning, we do not have the data labeled. We can say that we have the inputs, but not the outputs. So, the objective is to identify some pattern in our data. We can find groups or clusters that we think that belongs to the same group or output. Here we are necessary to obtain a model. Also, again, the objective we seek is to be able to predict the output given a new input.

Sentiment Analysis strengthens user value which helps users interacting with other users through reviews. These reviews and replies are used by the other users in deciding to go to the product or not. Since there are lots of existing approaches in sentiment analysis, such as Naive Bayes, Logistic regression in classifying the reviews, these approaches are effective but not in terms of accuracy. In order to enhance the accuracy, lot of research studies are going on in this field. In this paper, a specialized approach is utilized to increase the accuracy of the classification.

\section{Literature Review}

Table 1. Study of sentiment analysis

\begin{tabular}{|c|c|c|c|}
\hline S.no & Studies & $\begin{array}{l}\text { Algorithm / } \\
\text { Method Used }\end{array}$ & Description \\
\hline 1. & $\begin{array}{l}\text { Twitter part-of-speech tagging using pre- } \\
\text { classification hidden Markov model - } 2012 \text { (Sun } \\
\text { et al., 2012) }\end{array}$ & $\begin{array}{l}\text { Hidden Markov } \\
\text { Model }\end{array}$ & $\begin{array}{l}\text { It generally classifies the tweet as positive, negative } \\
\text { or neutral }\end{array}$ \\
\hline 2. & $\begin{array}{l}\text { Predicting Helpfulness Ratings of Amazon } \\
\text { Product Reviews - } 2012 \text { (Rodak et al., 2014) }\end{array}$ & $\begin{array}{l}\text { SVM and Naive } \\
\text { Bayes }\end{array}$ & $\begin{array}{l}\text { It covers automata features of data and also } \\
\text { classified based on token and syntactic analysis }\end{array}$ \\
\hline 3. & $\begin{array}{l}\text { Survey on Product Review Sentiment Analysis } \\
\text { with Aspect Ranking - } 2013 \text { (Patil and Mane, } \\
\text { 2016) }\end{array}$ & SVM & $\begin{array}{l}\text { Classification is done based on aspects of the } \\
\text { product. Every aspect are provided with aspect } \\
\text { ranking }\end{array}$ \\
\hline 4. & $\begin{array}{l}\text { Automatically detecting and rating product } \\
\text { aspects from textual customer reviews-2014 } \\
\text { (Bancken et al., 2014) }\end{array}$ & or & Special algorithm for aspect-based classification \\
\hline 5. & $\begin{array}{l}\text { Unsupervised Opinion Mining From Text } \\
\text { Reviews Using SentiWordNet }-2014 \text { (Soni and } \\
\text { Patel, 2014) }\end{array}$ & Sentiword Net & $\begin{array}{l}\text { The classification is done based on aspect level } \\
\text { which finds out aggregate scores for a particular } \\
\text { aspect (Fixed Syntactic patterns) }\end{array}$ \\
\hline 6. & $\begin{array}{l}\text { Ontology-based sentiment analysis of twitter } \\
\text { posts. Expert Systems with Applications - } 2015 \\
\text { (Kontopoulos et al., 2013) }\end{array}$ & $\begin{array}{l}\text { Ensemble } \\
\text { Approach }\end{array}$ & Classification is done based on polarity \\
\hline 7. & $\begin{array}{l}\text { Sentiment analysis using product review data. } \\
\text { Journal of Big Data - } 2015 \text { (Fang and Zhan, 2015) }\end{array}$ & Naive Bayes & $\begin{array}{l}\text { Extracting subjective content and tackling polarity } \\
\text { categorization problem }\end{array}$ \\
\hline 8. & $\begin{array}{l}\text { Sentiment Analysis for Movie Reviews }-2015 \\
\text { (Goyal and Parulaker., 2015) }\end{array}$ & Random Forest & $\begin{array}{l}\text { Classified by counting the number of words } \\
\text { repeated }\end{array}$ \\
\hline 9. & $\begin{array}{l}\text { User Bias Removal in Fine Grained Sentiment } \\
\text { Analysis - } 2016 \text { (Wadbude et al., 2016) }\end{array}$ & SVM & $\begin{array}{l}\text { Normalizing each user review score with respect to } \\
\text { mean and standard deviation of all products rated } \\
\text { by the user }\end{array}$ \\
\hline 10. & $\begin{array}{l}\text { A Twitter Sentiment Analysis for Cloud } \\
\text { Providers: A Case Study of Azure - } 2016 \text { (Qaisi } \\
\text { and Aljarah, 2016) }\end{array}$ & Naive Bayes & $\begin{array}{l}\text { AWS and Azure. The Opinion of customers around } \\
\text { each one of them }\end{array}$ \\
\hline
\end{tabular}

Shichang Sun and his team (Sun et al., 2012), used the classifier to classify the tweets as positive, negative and neutral, which uses POS (Parts of Speech) tagging for pre-processing of the twitter data. POS tagger is invoked as a pre-processing method is used in NLP program for information retrieval and extraction. POS tagging method uses sequential classification, which is a strategy of pre-classification. In the syntactic level task, an advanced approach of HMM model to analyze the result of sentimental subjectivity, i.e., POS tagging. The information on Subjectivity is explained as a pre-classified process in the model of interval type HMM. Procedure into the interval-type HMM. The degree of Subjectivity of the testing sentence is invoked as a combination factor to 
International Journal of Mathematical, Engineering and Management Sciences

Vol. 4, No. 2, 508-520, 2019

https://dx.doi.org/10.33889/IJMEMS.2019.4.2-041

select a suitable value from the interval. The Experimental analysis and results on datasets of public tagging show that the recommended strategy improvised the performance of POS tagging. The advantage of this method is to enhance the POS tagging performance than the other algorithms. It is better than the human classified training set. Pre-classification that helps in featuring training set models. The drawback of this method is it Can be utilized for only specified parameters and not on every other feature. It takes longer for processing than other types of algorithms. Slower when deciding the algorithms.

Jordan Rodak and his team (Rodak et al., 2014) developed a machine learning approach. After much deliberation and based on the quality of varying reviews, has said that they aimed to develop a machine learning approach to assess and rank the helpfulness of reviews automatically. Automatic classification helps to analyze poorly written reviews which lack competent information needed for the consumer. Classifier identifies and analyzes the patterns from the given data, and these patterns promise to facilitate upon review publishing.

In the paper of Patil and Mane (2016), it shows were identifying ranked aspects as per their importance. Free text reviews are viewed as input to our system. Free reviews are parsed using NLP which identifies the aspects of the particular product. For classifying sentiments supervised classifier SVM is used, then we applied probabilistic aspect ranking algorithm for natural aspect ranking. Advantages are it classifies very quickly. Accuracy is better than logistic regression. Disadvantages it works only on specified features. The ranking should be done properly, or else it leads to confusion.

Wouter Bancken and his team (Bancken et al., 2014) used an advanced algorithm called aspectator to analyze the aspect based sentiments that provided accuracy based on domains. Handcrafted dependency paths have been matched in the individual sentence to find opinions revealed towards candidate aspects. Then, it bundles the same aspect mentioned at different places by using a WordNet-based similarity measure. In the end, its analysis and brings out a sentiment score for each aspect, which identifies the overall emerging opinion of a group of customers towards an exclusive aspect of given the product. Domain-specific knowledge and seed word do not require in this approach since it only engages the self-sentiment lexicon. Advantages of this paper are the specialized algorithm that produces better performance and classification. Sentiment scores are calculated predictively and quite better than standard algorithms. Disadvantages of this paper are the accuracy is low for certain features and not suitable for all the features. Matching takes longer time than usual in aspect ranking.

Soni and Patel (2014) used the aspect-level sentiment analysis, which takes into an account that a document contains an opinion on multiple aspects of one or more objects in the document. The classification is done based on the aspect level, which finds out aggregate scores for a particular aspect. SentiWordNet uses the Senti-features to extract uses domain-specific features which can build a dictionary from abbreviation, slang words, and emoji in a message that is useful before extending the message with different features. The advantage of this paper has produced positive results in more aspects than other aspects. Feature extraction plays a crucial role and hence helps in the classification of SentiWordNet values. The disadvantage of this paper cannot classify the features with other algorithms. Accuracy varies for every other input.

Fang and Zhan (2015) has done the sentimental analysis on product review data which uses a Naive Bayes classifier for extracting subjective content and tackling polarity categorization problem. It 
International Journal of Mathematical, Engineering and Management Sciences

Vol. 4, No. 2, 508-520, 2019

https://dx.doi.org/10.33889/IJMEMS.2019.4.2-041

concentrates on both sentence-level and document level. A general process is proposed with adequate process descriptions to categories sentiment polarity. In this study, online product reviews on amazon dataset have been utilized for analysis. Both levels of experiments categorization such as sentence-level and state-level are conducted with encouraging results. The advantage of this paper is to provide medium accuracy when compared to SVM classifier, which helps the customer to make a quick purchase decision and this gives additional values to the classifier. The disadvantage of this method is a Naive Bayes does not produce satisfying accuracy, then algorithms like aspectator, sentiwordnet, etc. It cannot be recommended to the user for decision as the accuracy is not great.

Goyal and Parulaker (2015) used movie review on the basis of text using random forest classifier by counting the number of times the word repeated. "It can be used to determine the attitude of the reviewer concerning various topics or the overall polarity of review. Using sentiment analysis, the state of mind of the reviewer, while providing the review and understand if the person was "happy," "sad," "angry" and so on. Sentiment Analysis of a set of movie reviews given by reviewers and try to understand what their overall reaction to the movie was, i.e., if they liked the movie or they hated it. The relationships of the words in the review to predict the overall polarity of the review". The advantage of this method using Relative efficiency and easy implementation. Standard memorybased methods are its high accuracy and scalability. The disadvantage of this method demonstrates poor accuracy when there is little data about users' ratings. Systems are not content aware, meaning that information about items is not considered when they produce recommendations.

Rahul Wadbude and his team (Wadbude et al., 2016) used Fine-grained sentiment analysis of text reviews has recently gained much attention to the natural language processing community. Most works were done earlier focusses on creating efficient feature representations of the text reviews for classification. The methods generally ignore other common attributes like user identity, product identity, and helpfulness rating during fine-grained sentiment classification. A significant problem with current classification models is noise due to the presence of user bias in review ratings. The advantage of this paper, it predicts the score for a review as the mode of almost all reviews. Hyperparameters provide better performance. Produces consistent output for all testing input. The disadvantage of this paper, it works only on feature representation techniques. Classifying Unigram, Bigram makes difficult processing.

Qaisi and Aljarah (2016) stated that SNS are web-based activities which are based on friendship, acquaintances, interests, relationships, family, etc. This allows the participants to communicate on different topics purposes like sharing ideas, information, emotions, and events. In order to understand the current status of SNS, stakeholder and service providers had allowed researchers to use text-mining method in addition to sentiment analysis. Text mining is the process of extracting knowledge from available text online, whether on SNS, public forums or the internet generally regarding a certain topic. Text mining is the process of extracting knowledge from the available content of SNS online materials, and public forums are from the Internet on a specific topic. This method is utilized in the area of information retrieval extraction and natural language processing. In order to understand the customer opinions about Amazon, dataset extracts from their cloud products need to be analyzed. The advantage of this paper, it works well with any input and for all types of datasets. Classifying emoticons in a better way with better accuracy. Reliable storage of classified reviews in the cloud for more massive datasets. The disadvantage of this method, it cannot work well for sentiment net texts. Not works well for infrequently used words. Lesser accuracy of other data than emoticons. 
International Journal of Mathematical, Engineering and Management Sciences

Vol. 4, No. 2, 508-520, 2019

https://dx.doi.org/10.33889/IJMEMS.2019.4.2-041

The recommendation system produced by the proposed system was optimized using Sentiment Analysis with the help of Learning Automata (LA) by Krishna et al. (2013) The performance proposed system can be improved using Learning Automata which help a user to identify an accurate location based on the need. In relying on the previous feedback on the places queried by the user, the proposed system formulates the score analysis by using Sentimental Analysis (SA) and with these approach users' opinions are analyzed. The proposed system has been incorporated with a cloud platform. The advantage of this method is Ranked in decreasing order of probability of relevance. Helps in better recommendation system for the users to purchase products online. They are producing higher accuracy when compared to non-cloud platforms. The disadvantage is Implementation is challenging and difficult. Classification takes more time than usual. It is based on feedback on previous data and not on current working one.

In the existing system, there are so many algorithms are used in the classification of the reviews. These algorithms are providing lesser accuracy. Accuracy needs to be measured using various metrics. Classification of reviews is generally positive, negative or neutral. Hence, when the accuracy is very low, the particular algorithm unable to decide the review as a positive, negative or neutral one. These reviews are not able to use further for the recommendation. Time taken by each algorithm for classifying the data is low.

\section{Challenges}

Increasing accuracy is one of the significant challenges in sentiment analysis. Most of the algorithms can be able to classify the dataset but failed in producing accuracy. This leads to the system unable to recommend further. Here, implementing the ensemble approach made a significant challenge in combining the different algorithms. Each algorithm has different functionalities and works in their way. So, combining these algorithms and classifying the data done much work and also this voting algorithm (Ensemble) must provide higher accuracy than the existing system. The existing system produces lower accuracy, and the proposed approach must provide the accuracy more excellent than the existing system algorithms.

\section{Motivation}

Sentiment analysis is one of the trending research studies undertaken by various researchers. Currently, a lot of approaches, methods, tools, and techniques are evolved in this field to increase the performance and accuracy for the recommendation of the product or data. Online reviews differ from typical text documents in that they contain fewer features (i.e., they are short documents). Text reviews are generally consisting of various expressions, traditional words which are obtained through various researchers.

To identify the sentiment of the entire document, scoring algorithms can be used by classifying weighted phrases from the previous side by a proprietary way of adding them up. Here users get an opportunity to express their individual opinion about particular topics, and examples are product review sites, blogs, discussion forums, and social networks. Customers opinions can be expressed in various forms in any of these websites for example customer reviewing products in websites such as Amazon, app review sites, and tourist booking sites. which allow the rating of the product based on a fixed scale and personal opinions. Every algorithm is efficient in their ways. Every algorithm has various advantages and disadvantages by performance, accuracy, and quality of training the data sets. 
International Journal of Mathematical, Engineering and Management Sciences

Vol. 4, No. 2, 508-520, 2019

https://dx.doi.org/10.33889/IJMEMS.2019.4.2-041

Lots of researchers are doing in order to increase the accuracy of the data. This accuracy profoundly helps in the recommendation for the users who use the system. Accuracy is one of the essential measures in classification. This decides whether the algorithm functions properly or not for the dataset. Choosing an algorithm is highly depends on what kind of data we are using and how we are going to process and analyze it.

\section{Proposed Method}

Ensemble approach is the proposed method where the collection of algorithms is used in classification. Existing algorithms are combined and classified by taking the votes (mode values of the predictors). This is highly challenging as the process of creating a voted classifier is difficult since the concentration of classification is on getting a higher accuracy than the existing one. Mode values are taken for every algorithm. The ensemble is highly effective on supervised classification approaches because the labels are embedded along with the data set.

In the proposed system, an Ensemble approach is used to classify the review. Ensemble approach is also known as Majority voting or voting classifier. Ensemble approach is the combination of algorithms. One or more algorithms are classified, and the output is provided based on the voting of the predictors (mode values of all the algorithms). Here, Ensemble approach is used by combining Naive Bayes and SVM. Thus, the proposed method will produce higher accuracy when compared to the existing algorithms that produce lesser accurate than the proposed one. The ensemble method generally provides better accuracy as it combines the algorithms that help in functioning effectively. This method provides better performance than could be obtained from any of the algorithms alone. Every model predicts for each instance, and the final output prediction is the one that receives more than half of the votes. If none of the predictions get more than half of the votes, the ensemble method could not make a stable prediction for this instance.

Advantages

- Ensemble method produces higher accuracy than other algorithms which functions alone.

- It is generally used to generate multiple hypotheses using the same base learner.

- It is effective in combining with more than two algorithms and avoids overfitting.

- Combination of algorithms helps in functioning all the algorithms simultaneously rather than working it alone by reducing each other drawback.

- Ensemble method reduces variance, and they average out biases.

\subsection{Processing Proposed Modules}

\subsubsection{Retrieval Module}

In this module Figure.1, Training dataset will be created by collecting data using the Amazon API. A dataset can also be created using a scraping tool, and those are stored in excel. Data will be presented in a structured way, and there is no ambiguity in the data. The retrieved data may be stored in a file, printed, or viewed on the screen. Based on our system requirement dataset are collected as some of the datasets will be the huge size which we have to analyze those before using it. It is a supervised classification, and a dataset will contain labels for classification. Label values will represent data. 
International Journal of Mathematical, Engineering and Management Sciences

Vol. 4, No. 2, 508-520, 2019

https://dx.doi.org/10.33889/IJMEMS.2019.4.2-041

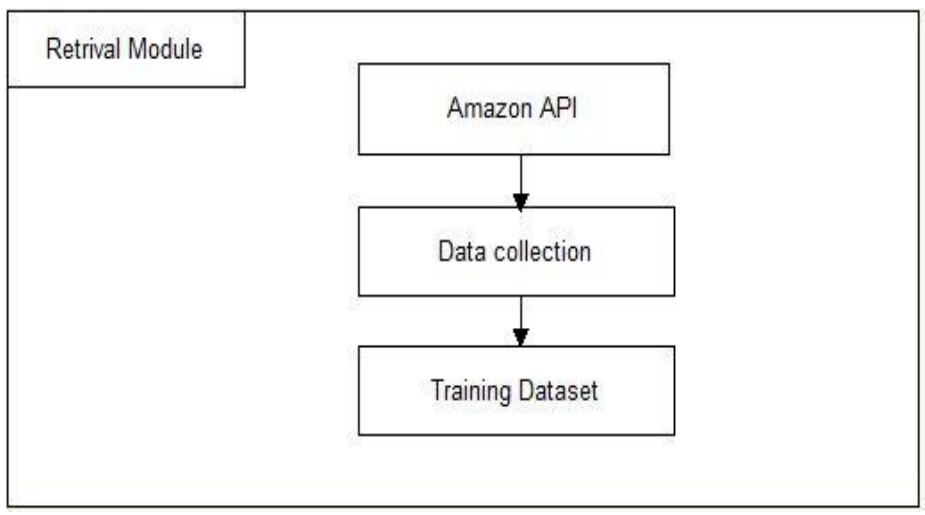

Figure 1. Retrieval Module

\subsubsection{Pre-Processing Module}

Once the data are collected, it should undergo pre-processing (Figure 2) in order to remove unwanted phrases, words, symbols. The representation and quality of data are first and foremost before running an analysis. Initially symbols, like @, hashtags\# (Stop Words) will be removed, followed by spell checking and emoticons tagging. The next phase proceeded with tagging POS (Parts Of Speech). Here, all the necessary POS words and phrases such as verbs, adverbs, and adjectives are extracted. These words will be considered with the weighted scores.

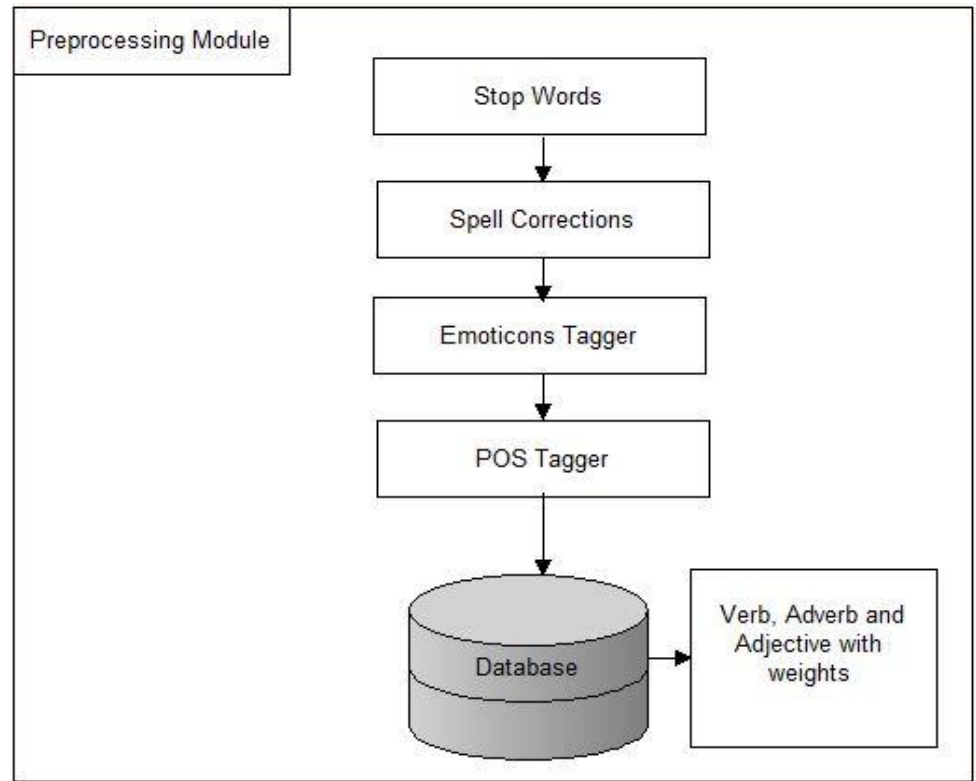

Figure 2. Pre-Processing Module 
International Journal of Mathematical, Engineering and Management Sciences

Vol. 4, No. 2, 508-520, 2019

https://dx.doi.org/10.33889/IJMEMS.2019.4.2-041

\subsubsection{Classification Module}

Here, Training data will be collected and classified (Figure 3) using Naive Bayes, SVM classifiers. Generally, there are two types of classification such as Supervised and Unsupervised. Supervised classification will be provided with labels. The training data is composed of a set of training examples whereas unsupervised classification will not be provided with any set of labels. We have proposed some supervised classifiers such as Naive Bayes and SVM. Naive Bayes is a simple technique for constructing classifiers to assign class labels for problem instances, represented as vectors of feature values, where the class labels are chosen from some finite set. By Support Vector Machine (SVM), the state is not directly visible, but the output dependent on the state is visible. Each state gets a probability distribution over the possible output tokens. Therefore, the sequence of tokens generated by an SVM gives some information about the sequence of states. Finally, an Ensemble approach is implemented by combining all the above methods. Once the classification is done, majority among them is voted (Accuracy), and the classified produces better accuracy than the existing system.

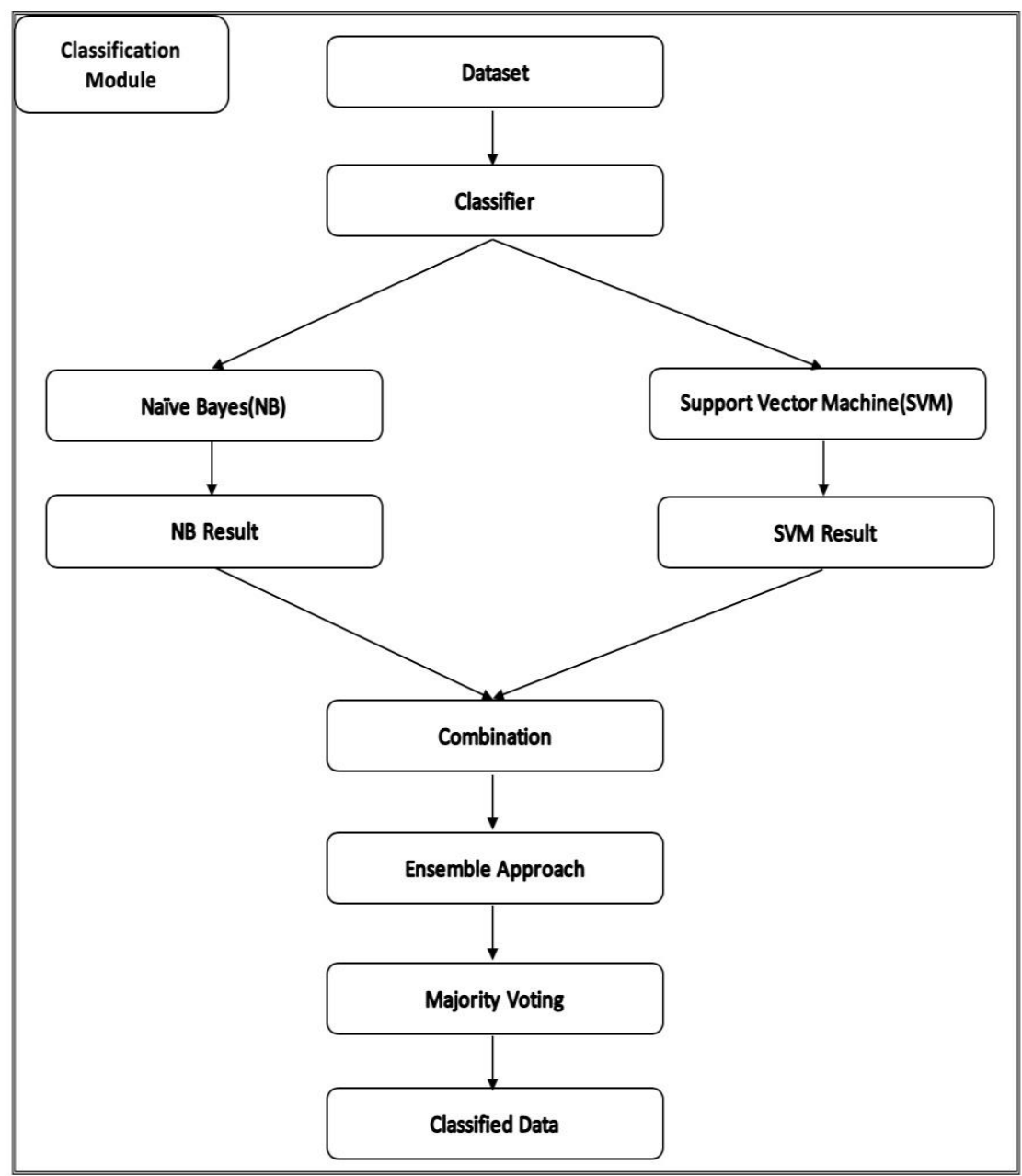

Figure 3. Classification Module 
International Journal of Mathematical, Engineering and Management Sciences

Vol. 4, No. 2, 508-520, 2019

https://dx.doi.org/10.33889/IJMEMS.2019.4.2-041

\section{Algorithm Implementation}

\subsection{Naive Bayes Algorithm}

A Naive Bayes classifier is a simple probability-based algorithm. It uses the Bayes theorem but assumes that the instances are independent of each other, which is an unrealistic assumption in practical world Naive Bayes classifier works well in complex real-world situations.

$P(H / X)=\frac{P(X \mid H) P(H)}{P(X)}$

- P stands for the probability of the variables within parenthesis.

- $\mathrm{P}(\mathrm{H})$ is the prior probability of marginal probability of $\mathrm{H}$ its prior in the sense that it has not yet accounted for the information available in $\mathrm{X}$.

- $\mathrm{P}(\mathrm{H} / \mathrm{X})$ is the conditional probability of $\mathrm{H}$, given $\mathrm{X}$ it is also called the posterior probability because it has already incorporated the outcome of event $\mathrm{X}$.

- $\mathrm{P}(\mathrm{X} / \mathrm{H})$ is the conditional probability of $\mathrm{X}$ given $\mathrm{H}$.

- $\mathrm{P}(\mathrm{X})$ is the prior or marginal probability of $\mathrm{X}$, which is normally the evidence.

It can also be represented as. Posterior=likelihood $*$ prior normalising constant The ratio of $\mathrm{P}(\mathrm{X} / \mathrm{H}) / \mathrm{P}(\mathrm{X})$ is also called a standardized likelihood.

\section{STEPS:}

Step 1: Split data into k subsets of equal size.

Step 2: Use each subset in turn for testing, the remainder for training.

Step 3: Find the total positive and total negative probability of the data.

Step 4: For every word in a dataset, finds its positive probability and negative probability.

Step 5: Multiply all the positive and all the negative probability values.

Step 6: Multiply those values with the total probability values.

Step 7: Select the sentiment for which probability has more significant value.

\section{Support Vector Machine}

SVM is used in regression analyzing, and classification for a set of training sets with different forms and categories. Calculate the similarity from only a subset of training points, which we compute in the training phase. So, in the training phase, we fix a subset of training points from which we compute the similarity of any test point. These selected training points are called support vectors since only these points will support our decision of selecting the class of a test point. We hope that our training phase finds as few as support vectors so that we have to compute the fewer number of similarities. Now, once we have selected support vectors, we assign a weight to each support vector, which tells how much importance we want to give to that support vector while making our decision.

\section{STEPS:}

Step 1: Find weight for each training point, and those points whose weight becomes zero are not support vectors i.e. their importance is zero during test time, and rest are support vectors.

Step 2: Transform each of the vectors into some other higher dimensional vector, and then take dot product between those two complex higher-dimensional support vectors.

Step 3: Find the maximum of vectors as a sentiment value. 
International Journal of Mathematical, Engineering and Management Sciences

Vol. 4, No. 2, 508-520, 2019

https://dx.doi.org/10.33889/IJMEMS.2019.4.2-041

\section{Ensemble Method}

The proposed methodology is focused on combining Naive Bayes and Support Vector Machine classification algorithms to enhance the results. In the figure: 3 , it is presented with an ensemble algorithm which shows us principle of data processing from dataset up to obtaining the results. Training and testing data had been preprocessed and cleaned before it was passed as the input of machine learning algorithms. Ensemble approach is used to classify the review. Ensemble approach is also known as Majority voting or voting classifier. Ensemble approach is the combination of algorithms. One or more algorithms are classified, and the output is provided based on the voting of the predictors (mode values of all the algorithms). Here, Ensemble approach is used by combining Naive Bayes and SVM. Thus, the proposed method will produce higher accuracy when compared to the existing algorithms that produce lesser accurate than the proposed one. The ensemble method generally provides better accuracy as it combines the algorithms that help in functioning effectively. This method provides better performance than could be obtained from any of the algorithms alone. Every model makes a prediction analysis at each instance, and the final output prediction is the one that receives more than 50 percent of the votes. If none of the predictions get more than half of the votes, the ensemble method could not make a stable prediction for this instance.

\section{STEPS:}

Step 1: Implement existing algorithms such as Naive Bayes and SVM.

Step 2: Create a class called Voting classifier and inherit the list of classifiers used such as Naive Bayes and SVM.

Step 3: Classification of the training set based on this voting classifier and this would calculate the votes for every algorithm that classifying the dataset.

Step 4: In voting classifier class, create our own classify method. Now iterating through our list of classifiers objects. Then, for each one, we ask it to classify based on the features.

Step 5: After we are done iterating, we then return the mode (votes), which is just returning the most popular vote.

\section{Dataset Analysis \\ 8.1 Testing Methods}

Most of the time we will want to do random sampling to obtain training and test sets that are more or less representative population samples. However, we should be aware of issues like class imbalance where for example the frequency of one class dominates in our target values. In such cases, we probably have to do stratified data splitting based on the classes for our test and training set to have the same proportion of both classes. Hence, the model is trained using a training dataset and tested using testing dataset for its accuracy.

\subsubsection{Training and Testing Data}

Training data is the data on which the machine learning programs learn to perform correlational tasks (classify, cluster, learn the attributes). Here, training data consists of feature sets of 3996 positive and 3994 negative data which has been taken from the original dataset. These data consist of reviews of the particular product. The text review is a mixture of emoticons, stop words, punctuations such as comma, apostrophe, exclamatory, etc. These values will be pre-processed in order to get the better result. The training dataset will be trained using Naive Bayes classifier, and further will be tested using testing data for classifier's accuracy. The training dataset is the most 
important dataset for the classification. Testing data is the data, whose outcome is already known (even the outcome of training data is known) and is used to determine the accuracy of the machine learning algorithm, based on the training data (how effective the learning happened). Here, testing data consist of remaining data left from the training data set. The testing data consist of 3996 positive and negative text reviews. These test data will be used as input to check the result of the input as positive or negative to recommend that particular product. Testing data is the one that checks for the effectiveness and accuracy of the algorithm on which how well the algorithm classifies the data and process the output.

\section{Results and Discussion}

We were monitoring the process of classification and how the data set was pre-processed, classified and produced the accuracy. The data set was pre-processed, then classified using Naive Bayes, SVM, Ensemble method. Then, the accuracy was calculated for every algorithm. Once the accuracy is calculated, the accuracy is compared with all algorithms and display it through the plotted graph. The interface (Figure 4.) is created using python, and the output is displayed as positive or negative based on the review the user type from the interface.

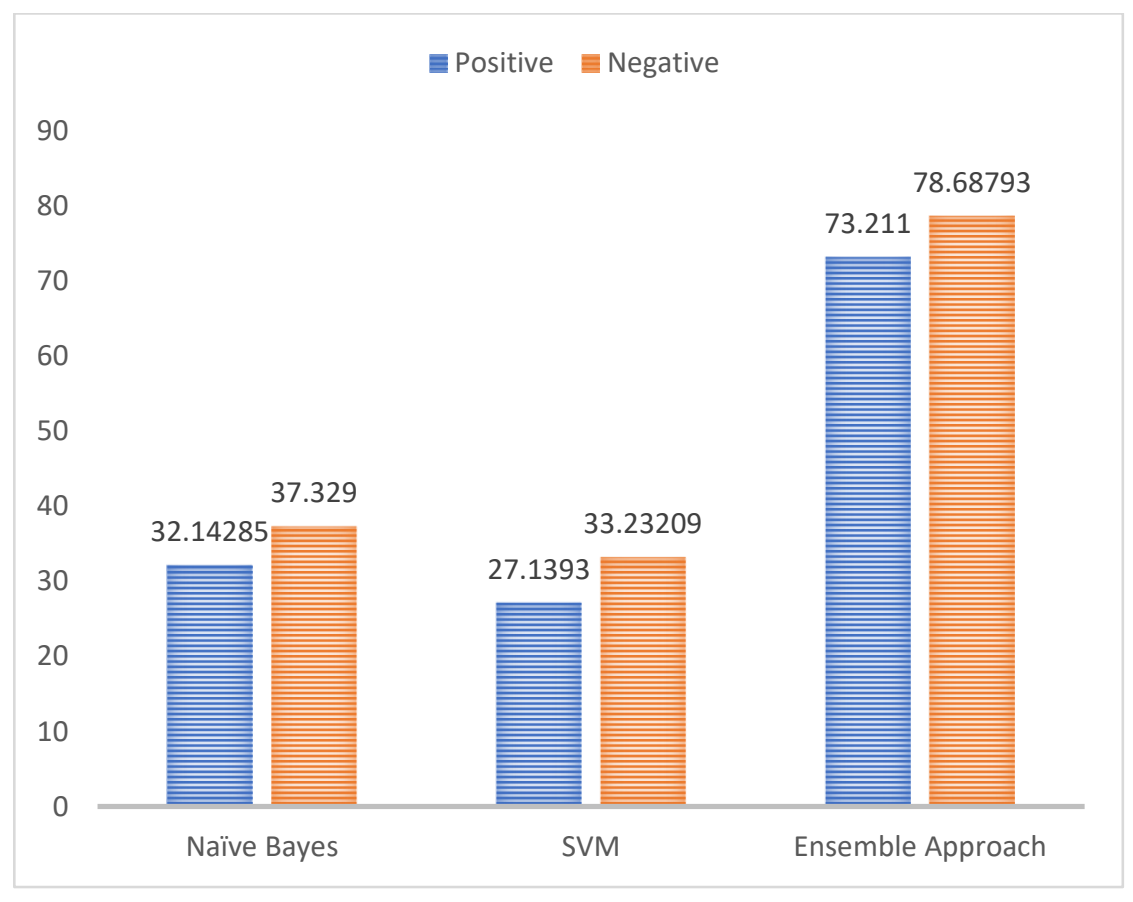

Figure 4. Analysis of data classification

\section{Conclusions and Future Work}

The proposed an Ensemble method that helps in providing better accuracy than the current existing algorithm. We also effectively tackled the issue of performance, accuracy, speed execution of the algorithm. The proposed system produces better accuracy than the existing system. However, the 
International Journal of Mathematical, Engineering and Management Sciences

Vol. 4, No. 2, 508-520, 2019

https://dx.doi.org/10.33889/IJMEMS.2019.4.2-041

accuracy level varies with the total number of the classifiers we are combining to get a predicted output of the review. In this paper, we have proposed a method in order to increase the accuracy of the review classification. If the accuracy is higher, we can use the system for the recommendation for the users.

Further work will be based on the aspect level classification. Aspect level classification is something where the review will be specified rather than a standard classification. In aspect level classification, Features are specified and processed for the algorithms. For example, in the product review dataset, specified datasets such as electronics, books, mobile phones are taken, preprocessed and classified to get deeper accuracy, better performance. Thus, the review-based classification is highly developed these days and helps in the recommendation of the product to the users based on the reviews expressed by the user. We can also work on expressing emoticons used by the users in the review.

\section{Conflict of Interest}

All authors have contributed equally to this work. The authors declare that there is no conflict of interest in this publication.

\section{Acknowledgment}

The authors acknowledge and express the gratitude to Amazon online for providing the dataset for this paper.

\section{References}

Bancken, W., Alfarone, D., \& Davis, J. (2014, August). Automatically detecting and rating product aspects from textual customer reviews. In Proceedings of the 1st international workshop on interactions between data mining and natural language processing at ECML/PKDD, (pp. 1-16).

Fang, X., \& Zhan, J. (2015). Sentiment analysis using product review data. Journal of Big Data, 2(1), 1-14.

Goyal, A., \& Parulekar, A. (2015). Sentiment analysis for movie reviews. Available at https://cseweb.ucsd.edu/classes/wi15/cse255-a/reports/fa15/003.pdf.

Kontopoulos, E., Berberidis, C., Dergiades, T., \& Bassiliades, N. (2013). Ontology-based sentiment analysis of twitter posts. Expert Systems with Applications, 40(10), 4065-4074.

Krishna, P. V., Misra, S., Joshi, D., \& Obaidat, M. S. (2013, May). Learning automata based sentiment analysis for recommender system on cloud. In Computer, Information and Telecommunication Systems (CITS), 2013 International Conference on (pp. 1-5). IEEE.

Patil, H., \& Mane, P. M. (2016). Survey on product review sentiment analysis with aspect ranking. International Journal of Science and Research, 5(9), 749-752.

Qaisi, L. M., \& Aljarah, I. (2016, July). A twitter sentiment analysis for cloud providers: a case study of Azure vs. AWS. In Computer Science and Information Technology (CSIT), 2016 th International Conference on (pp. 1-6). IEEE.

Rodak, J., Xiao, M., \& Longoria, S. (2014). Predicting helpfulness ratings of amazon product reviews. Available http://cs229.stanford.edu/proj2014/Jordan\%20Rodak,\%20Minna\%20Xiao,\%20Steven\%20Longoria,\% 20Predicting\%20Helpfulness\%20Ratings\%20of\%20Amazon\%20Product\%20Reviews.pdf 
International Journal of Mathematical, Engineering and Management Sciences

Vol. 4, No. 2, 508-520, 2019

https://dx.doi.org/10.33889/IJMEMS.2019.4.2-041

Soni, V., \& Patel, M. R. (2014). Unsupervised opinion mining from text reviews using sentiwordnet. International Journal of Computer Trends and Technology, 11(5), 234-238.

Sun, S., Liu, H., Lin, H., \& Abraham, A. (2012, October). Twitter part-of-speech tagging using preclassification Hidden Markov model. In Systems, Man, and Cybernetics (SMC), 2012 IEEE International Conference on (pp. 1118-1123). IEEE.

Wadbude, R., Gupta, V., Mekala, D., Jindal, J., \& Karnick, H. (2016). User bias removal in fine grained sentiment analysis. In European Chapter of the Association for Computational Linguistics, arXiv:1612.06821v1 [cs.CL] 20 Dec 2016. 